\title{
Oncoplastic Breast Surgery in India: Thinking Globally, Acting Locally
}

\author{
Chaitanyanand B. Koppiker ${ }^{1} \cdot$ Chintamani $^{2}$. Santosh Dixit ${ }^{1}$
}

Published online: 24 April 2019

(C) Association of Surgeons of India 2019

\section{Background}

The field of breast surgery has undergone significant evolution since the emergence of Halstedean concept of total mastectomy (TM) [1]. In the last three decades, breast conversation therapy (BCT) which involves breast conservation surgery followed by radiation therapy (RT) has gained acceptance as a standard of care $[2,3]$. In recent past, more emphasis has been laid on the esthetics of breast surgery as increasing evidence indicates that a bad cosmetic result can translate to significant depression and worse quality of life (QoL) outcomes [4, 5].

The rapidly advancing field of oncoplastic breast surgery (OBS) offers a pragmatic alternative to TM and BCT. OBS is based on the fundamental tenet that the breast is not only a functional but also an esthetic organ. Thus, OBS principles are based on integrating the principles and practices of conventional breast onco-surgery with those of plastic surgery [6]. OBS aims to achieve uncompromised oncological safety, better esthetic, and psycho-social outcomes that improve the QoL and prognosis in breast cancer patients $[7,8]$.

In the mid-1990s, Professor Werner Audretsch from Germany did pioneering work in OBS which then gained acceptance amongst European surgeons most notably in France, Italy, and the UK [9]. Subsequently, many breast and plastic surgeons from different parts of the world have contributed to the field via development of innovative surgical techniques and accessories, clinical management concepts, patient-reported outcome measures (PROMs) and QoL assessments [10-12]. Many breast surgeons from the UK took

Chintamani

drchintamani7@gmail.com

1 Orchids Breast Health Clinic, 1-2, Kapilvastu, Senapati Bapat Road, Pune, Maharashtra 411016, India

2 Vardhman Mahavir Medical College and Safdarjung Hospital, New Delhi Near AIIMS Hospital, Ansari Nagar, New Delhi, Delhi 110029, India initiatives in developing newer concepts in OBS which changed the landscape of this emerging field. Through national implementation under the NHS, OBS gained more acceptance in the medical fraternity in particular and in the society in general [13].

Internationally, OBS is now considered as a standard of care in the personalized management of breast cancer [14]. Based on the clinico-pathology and psycho-social profile of the patient, the oncoplastic surgeon will offer the following OBS options, namely:

(a) Level I/II oncoplasty: BCT with surgical procedure based on either volume displacement/rearrangement (i.e., local tissue rearrangement, mastopexy, reduction mammoplasty, extreme mammoplasty) or volume replacement (i.e., autologous fat grafting, transposition/ rotational flaps) [15]

(b) Level III/IV oncoplasty: Mastectomy followed by oneor two-stage breast reconstruction (i.e., expander or implant) or autologous breast reconstruction (i.e., abdominal tissue (DIEP, TRAM), latissimus dorsi flap, gluteal tissue, or other tissue sources) [16]

It is now well-established that OBS techniques provide optimal oncological safety, survival advantages, superior esthetic outcomes, and improvement in QoL of patients [17]. The OBS field is constantly evolving as a result of parallel developments in related fields of surgical tools and technology, lipo-filling, acellular dermal matrices (ADMs), breast implants, and regenerative scaffolds. In several developed countries, OBS has gained acceptance in routine medical management of breast cancer as the cost of OBS is covered under social insurance schemes.

In future, OBS will remain the most prominent trend in the field of breast surgery as is evident from the significant increase in number of original research articles, book chapters, clinical practice guidelines, conferences, educational curricula, and hands-on training workshops all over the globe [18]. In this milieu, it is only timely and pertinent that we try to understand the landscape of OBS in India. 


\section{Breast Cancer Scenario in India}

According to WHO classification, India represents a developing, low-to-middle income country (LMIC). Despite a few commonalities, the breast cancer profile in India is uniquely different than other developed or developing countries with reference to its epidemiology and clinico-pathology as well as clinical management strategies. According to WHOGLOBOCAN data, breast cancer is the most common cancer in India [19]. In 2018, 162,468 new breast cancer cases (14\% of all cancer cases) and 87,090 deaths (12.11\% of all cancerrelated deaths) were reported in India [19]. Even though women from all socio-economic strata are affected young (mid-forties), premenopausal urban women appear to be at high risk. Lack of awareness in Indian women about disease symptoms, screening modalities, self-breast examination, and/or routine mammographic screening has been attributed to negligence, thereby, resulting in costly delay in diagnosis and treatment.

For breast surgery, TM remains the most common option in many tier II and III cities. Only a few reputed tertiary cancer care centers in tier I cities representing public and private sector with adequate supporting medical infrastructure offer the BCT or OBS options. ADMs which are commonly used accessories for breast reconstruction are expensive and not available in India. In the absence of well-designed, comprehensive training programs, there is a serious paucity of skilled breast surgeons and allied health professionals appropriately trained in OBS. A vast majority of Indian population has no access to medical insurance. While central government employees are covered by the Central Government Health Scheme (CGHS), majority of affording patients in India opt for medical insurance from private sector. However, a major misconception amongst these insurance providers is that OBS is a cosmetic surgery and not part of routine clinical management of breast cancer. Consequently, insurance claims for OBS surgery are routinely denied, thereby, limiting its availability to the needy patients. As a result, OBS is mainly accepted by the affording patients from the upper echelons of the society who can opt for out-of-pocket payment for OBS procedures. However, it is noteworthy that the CGHS has now started providing limited financial coverage for few OBS procedures. Furthermore, there exists a serious lack of awareness about OBS not only in the medical fraternity but also in the society at large. The cancer patient advocacy groups have not been adequately sensitized to the issues pertinent to patient rights and poor uptake of OBS in India.

These challenging circumstances in India also provide opportunities to alter the OBS status quo by upgradation of OBSthemed surgical services, creating training programs and undertaking research in the local context. Recognizing the need of the hour, many eminent breast surgeons in India have now started including OBS in their surgical armamentarium. In this regard, the multi-disciplinary team at our breast units with support from collaborators has been actively involved in the last half-decade in propagating OBS in the Indian medical fraternity as well as the general public.

\section{OBS in India: a Reality Check}

In this article, we attempt to present few real-life scenarios related to OBS which are relevant to the Indian context:

\section{1. "One Oncoplastic Breast Surgeon-Dual Role" Model}

In the mid-1990s, delivery of OBS in the UK, North America, and Europe was at a minimum as there were very few surgeons who had acquired the dual skills of onco-surgery and esthetic surgery. Several oncoplastic units in Europe and North America functioned as a team of an onco-surgeon and plastic surgeon operating on the same patient in a step-wise manner [20]. In this model, the surgical skills of both surgeons were independently leveraged for optimal oncoplastic outcomes. However, the UK model of OBS delivery has shown that the same breast surgeon can be effectively trained for both types of surgeries effectively [13]. This model is also reminiscent of the evolution of oral cancer surgeons who also embraced the reconstruction surgeries along with cancer surgery of the oral cavity.

However, in a developing country such as India, the OBS field is still nascent and there is a scarcity of plastic surgeons who can work along with breast surgeons. Hence, this scenario necessitates the adaptation of the "one surgeon-dual role" concept, wherein a well-trained breast oncoplastic surgeon performs both the onco-surgery and esthetic procedures. From our experience, this model has proven to be costeffective as it reduces the double expenditure on surgeon's fees and ensures better post-surgical outcomes since the entire case is handled by the same surgeon. This arrangement also ensures better acceptance of OBS by patients due to the costadvantage as well as same surgeon handling the entire surgical protocol. Indeed, going forward, Indian OBS units could be modeled in a way that basic level I/II OBS techniques could be handled by a team of breast and plastic surgeons owing to the high case loads. However, cases requiring advanced level III/IV OBS techniques could be handled by a well-trained and experienced OBS surgeon. The biggest challenge for these models to be successful in India is the paucity of breast surgeons who are also oncoplastically trained.

\section{OBS for Indian Patients}

Several reports indicate that majority of breast cancers in India are diagnosed at an advanced stage (III or IV) [19]. This late-stage diagnosis has been a main reason for India having the dubious distinction of the highest incidence-to-mortality 
ratio in the world. As a result, clinical management protocols that employ neo-adjuvant chemotherapy (NACT) are most common. These scenarios also imply that total mastectomy would be preferred and rate of breast conservation will be less. Thus, the need for standard OBS procedures following mastectomy and well-justified surgical modifications for Indian patients will be even more relevant.

\subsection{Extreme Oncoplasty}

Due to advanced stage detection, it is common to identify multi-centric, multi-focal tumors in Indian patients. In such situation, total mastectomy is surgery of choice. However, for such scenarios, a novel OBS technique termed "extreme oncoplasty (EO)" has been developed that can conserve the breast and avoid total mastectomy [21, 22]. Indeed, in our study cohort of 40 Indian patients, we have been able to demonstrate that this technique is safe and feasible for the surgical management of multi-centric, multi-focal tumors [23]. Furthermore, the EO-operated patients will undergo standard of care RT, thereby completing the BCT protocols. The EO surgery is an excellent example for the Indian context that demonstrates how OBS can avoid unnecessary mastectomies by conserving the breast and offer better survival advantage associated with BCT.

\subsection{Therapeutic Mammoplasty}

Many Indian breast cancer patients present with advanced tumors diagnosed in large breasts which get frequently mastectomized. Several reports generated from the Therapeutic Mammoplasty (TEAM) study protocol indicate that therapeutic mammoplasty is an effective OBS procedure for breasts with large size, ptosis, presence of tumor in superior medial quadrant, or those carrying scars from prior surgery [24]. In obese patients, therapeutic mammoplasty has been shown to reduce the rate of margin positivity, re-excision rates, margin excision rates, and RT-associated complications [25]. Better cosmesis and satisfactory PROMs have been observed in such patients after the use of therapeutic mammoplasty. We have been able to demonstrate the feasibility of this OBS technique in the removal of the tumor from a large breast along with superior surgical outcomes by breast reshaping, upliftment, and size reduction as well as contralateral symmetrization (unpublished data; $n=190$ cases)

\subsection{Breast Reconstruction}

If indicated, many breast cancer patients are advised total mastectomy followed by immediate breast reconstruction (IBR) either with the help of a breast implant or autologous tissue. IBR improves QoL, overcomes mastectomy-associated depression, provided better self-esteem and femininity, and increases overall survival $[20,26]$.
In the west, a single- or two-stage breast reconstruction with implants/tissue expanders or autologous tissue (LD or DIEP flap) is a standard procedure [20, 26]. Implant-based breast reconstruction is mostly preferred as it improves cosmesis, requires single-step surgery, has shorter recovery time, and is cost-effective since both onco-surgery and reconstruction are performed by a single oncoplastic breast surgeon [27]. ADMs are commonly used surgical accessories derived from immune-compatible biological tissues or synthetic polymeric meshes that provide an additional mechanical support the implant in situ in the lower pole of the breast pocket [28].

Close to $70 \%$ of cases in the UK undergo pre-pectoral breast reconstructions with implant and ADMs indicating the popularity of this technique [29]. Autologous reconstructions using various breast flaps provide better post-surgery outcomes and cosmesis but are less popular due to issues such as donor morbidity, slower recovery time, and ancillary surgical care [29].

In India, the acceptance of implant-based IBR is very poor $(\sim 5 \%)$. Therefore, concerted efforts need to be taken up to change this scenario such as:

(a) Surgical cost-reduction by purposeful deployment of the "one-surgeon, dual-role" model.

(b) Creating affordable breast reconstruction packages which can absorb the implant cost.

(c) ADMs or synthetic meshes are not yet available in the Indian market. If available in future, their use in breast reconstruction in Indian patients is doubtful given their intrinsically high cost that will add up significantly to the overall surgical package.

This challenge also provides an opportunity to OBS surgeons to innovate in the surgical techniques in the lowresource settings LMIC settings such as India. For example, we have successfully demonstrated that a one-stage breast reconstruction with breast implants is feasible if aided by such novel surgical modification that employs autologous lower dermal skin (ALDS) derived either from the breast pocket or lower abdominal/thoracic skin [30]. With this technique, we have observed that even surgically challenging non-ptotic/ small breasts can be successfully reconstructed using implants without the need of ADMs (unpublished data):

(d) Level I OBS technique using extended LD flap has shown excellent results in breast reconstruction with any major post-surgery complications. This technique needs to be popularized in Indian OBS surgeons.

(e) One of the major impediments in the mainstreaming of implant-based IBR has been the rate of complications post-surgery and/or post-RT [31]. Moreover, even higher rates of such complications were observed after the use of ADMs or synthetic meshes [32]. 
In the Indian context, a two-stage breast reconstruction is not feasible due to poor patient acceptance. It is likely that implant-based reconstructions will perhaps remain the method of choice in Indian patients due to the problems associated with autologous reconstructions. Thus, the OBS community will need to collaborate with the RT community in designing integrated OBS and RT protocols to manage such scenarios. It should be noted that the available data on RT-induced complications after IBR has been generated using decade-old technology. Going forward, with more sophisticated RT equipment coming into picture, better planning of RT regimens and targeting dosing is possible, which is expected to reduce the RT-associated complications.

In addition, this RT-related problem also necessitates innovations in OBS surgical technique. Indeed, in our study cohort, the ALDS procedure was found to offer a protective advantage against RT-associated complications which are common in implant-based breast reconstructions [30]:

(f) Non-availability of ADMs or synthetic meshes in India is also an opportune challenge for polymer/material scientists and biomedical engineers. Newer biocompatible polymers, regenerative scaffolds, and 3-D-printed biomaterials are the paving the way for novel surgical innovations even in other disease domains [28]. Thus, the needs of OBS in low-resource settings can possibly foster research and development of novel, cost-effective biomaterials with a vision for entrepreneurship and global commercialization.

3. Integration of Multiple Oncology Domains with Breast Oncoplasty

The advent of newer principles of OBS is providing new avenues for multi-disciplinary collaboration between breast surgeons, radiologists, medical oncologists, radiation oncologists, geneticists, and psycho-oncologists. Indeed, the need for a regular multi-disciplinary tumor board in a breast oncoplastic unit cannot be overstated. Furthermore, oncology professionals in India are encouraged to embrace these developments in breast oncoplasty and design appropriate capacity building initiatives for honing their skills.

\subsection{Breast Radiology}

The placement of radiopaque clips in patients who have been advised NACT and BCT has been associated with better local control independent of stage and other clinicopathologic findings [33]. Therefore, placement of tumor-marker clips should be an integral part of the multi-disciplinary approach in appropriate patients undergoing OBS. The radiology team working in tandem with a breast oncoplastic unit should be adequately trained to undertake imaging of breasts in various situations such as prior to, during NACT phase, post-surgery and during long-term follow-up. Particular emphasis should be placed on imaging of reconstructed breasts (either with autologous or implant-based procedures) with the appropriate radiological modalities. In high-risk individuals identified by genetic testing, radiological analysis of breast density will be an additional decision-making tool that will be helpful to the breast surgeon in the choice of prophylactic risk-reducing surgery options.

\subsection{Breast Cancer Genetics}

NCCN has recommended multi-gene panel testing for germline mutations in hereditary breast and ovarian cancer (HBOC)-causing genes in breast cancer-affected patients or unaffected healthy individuals with a strong family history [34]. As recommended by ACMG, the multi-gene panel should include BRCA1/2 and several other genes which have been shown to contribute to the HBOC risk [35]. Given the affordable costs and logistics, such genetic testing is gaining widespread acceptance in major cities in India. Most major breast units now either have an in-house genetic clinic or regularly refer appropriate cases to genetics professionals for appropriate advice.

Very few breast surgeons in India are well-trained in the application of various OBS techniques in risk-reducing surgeries for high-risk individuals identified by genetic testing. These techniques include bilateral prophylactic mastectomy followed by reconstruction (for healthy, unaffected individuals) and contralateral prophylactic mastectomy followed by reconstruction (for index patients). For index patients, three real-life scenarios can be envisaged as follows: (i) genetic testing concurrent to primary diagnosis, (ii) genetic testing during disease-free interval, (iii) genetic testing after ipsilateral or contralateral recurrence. From 2015 onwards, genetic testing in India has gained momentum. Hence, it is expected that retrospective genetic testing of index patients during their disease-free intervals will be most common scenario. In addition, cascade genetic testing in the family members of the index patient with a specific pathogenic mutation is expected to identify high-risk, asymptomatic individuals. Our observations indicate that such individuals are keen to learn about their cancer risk mitigation options inclusive of prophylactic breast surgeries.

In these fast-evolving scenarios, it is incumbent upon the breast oncoplastic surgeon to be conversant about the HBOC germline testing and its applications in addition to the OBS repertoire. Various breast oncoplasty skill-sets and a pragmatic thought process for choosing the appropriate timing of surgery will have to be acquired. In addition, the breast surgeons will have to hone their counseling skills for the individuals as well as the family members.

Concurrently, these advances justify the important role for genetic counselors in breast oncoplastic units. These 
professionals can create significant impact during pre- and post-test counseling of individuals and their families about the risk assessment process [36]. In addition, they can assist the breast surgeon in conveying the various intricacies of riskreducing surgeries to the individuals so as to ensure better acceptance of the surgical options deemed fit.

\subsection{Medical Oncology}

Several reports indicate that NACT-guided downsizing of the breast tumor provides an opportunity to the surgeon for breast conservation when total mastectomy is obvious [37]. Therefore, when indicated, the success of a well-designed NACT plan will influence the outcomes of OBS. As a result, the breast oncoplastic surgeon will have to work in tandem with a well-trained and experienced medical oncologist who is familiar with the fast-evolving breast cancer NACT concepts. In this regard, joint counseling sessions with the patients that involve the oncoplastic breast surgeons and medical oncologists should be encouraged to discuss the plan of treatment.

\subsection{Breast Radiotherapy}

If indicated, RT is integral to breast cancer management for effective local control and reducing the risk of recurrence. Similar to the global scenario, standardized RT after breast conversation surgery is now well-established in major breast centers in India. However, RT after breast oncoplastic surgery is not so common yet due to the paucity of dedicated breast oncoplastic units. In general, RT protocols may have to undergo appropriate modification for patients who have undergone OBS. This is particularly true in the case of autologous or implant-based reconstructions since the choice of RT technique, dosimetry, and duration will determine the post-RT complications. Indeed, a higher rate of post-RT complications such as fibrosis and capsular contracture which may sometimes lead to implant failure has been reported in patients who have undergone implant-based reconstructions [38]. We have previously shown that the ALDS-based implant reconstructions followed by careful planning of RT protocol can reduce the rate of such complications [30]. In parallel, our RT team has innovated to incorporate a SimultaneousIntegrated Boost (SIB)-IMRT protocol for patients with multi-centric/multi-focal tumors who have undergone extreme oncoplasty [23]. As the number of OBS procedures are expected to increase in India, the RT professionals will have to adapt and integrate such innovative RT protocols into their armamentarium.

\subsection{Breast Psycho-Oncology}

In the currently popular theme of mind-body medicine, OBS is an appropriate fit. Post-mastectomy depression is a common and negative regulator of treatment outcomes and disease prognosis [4]. When given a choice of treatment options, the patients' acceptance and decisionmaking is largely influenced by her psychological state of mind. Several studies have indicated that OBS improves patients' psycho-social profiles, self-esteem, quality of life, and overall confidence [4, 5]. Long-term follow-up studies in patients who have undergone OBS have indicated a survival advantage [39].

However, in the predominantly patriarchal Indian family set-up, the voice of the breast cancer patient is seldom heard. The middle-aged Indian woman is the fulcrum of the entire family who happily caters to the personal needs of her parents, in-laws, husband, and children while sometimes sidelining her own personal and professional needs. In our experience, many Indian women with such psyche, if and when faced with a breast cancer diagnosis, undergo acute and severe depression. They are either unaware of or disregard such important personal issues related to in self-esteem, body image, and sexual well-being in light of a possible mutilating breast removal surgery. At such junctures, the role of a psychological counselor is vital to help the patient express her anxieties and understand the treatment options designed in her best interest. Given these India-specific scenarios, most breast oncoplastic units should have psycho-oncology counselors as an integral part of their units. Furthermore, the breast oncoplastic surgeons should ensure to impart appropriate domain knowledge to these professionals.

One of the criteria utilized for critical assessment of the success of breast oncoplasty is patient-reported outcome measures (PROMs). BREAST-Q is a validated questionnaire used commonly in this field for collecting PROMs data at stipulated post-surgery intervals (ranging from 1 to 5 years) [40]. PROMs are a routinely used tool in breast oncoplastic units all over the world for clinical audits as well as research. The presence of a psycho-oncology counselor in such units facilitates the BREAST-Q interview and PROMs data collection. Indeed, our psychooncology team has demonstrated the utility of collecting yearly PROMs for assessment of patient acceptance of OBS procedures [41].

\section{Need for OBS Training in India}

To realize the true potential of OBS in breast cancer management in Indian patients, there is an exigent need to create a well-trained work force of breast surgeons, radiologists, medical oncologists, radiation oncologists, geneticists, psychology counselors, and nurses who are aligned to the its ever-evolving principles and practices. Successful OBS training programs have been created in many countries [42, 43]. To this effect, senior breast 
oncoplastic surgeons from India and the UK have created a structured training program from aspiring breast oncoplasty surgeons [www.breastoncoplasty.org]. This multi-disciplinary program has been designed to provide the trainees in-depth understanding of not only OBS principles but also about breast cancer biology, molecular oncology, radiology, pathology, clinical management, and research. The OBS training involves deliberations on level I and II oncoplasty along with various OBS surgical techniques enlisted above. Hands-on training is provided via surgical simulations, live mark-up sessions, and operative experiences under the mentorship of experts from various oncoplastic domains. Periodic training workshops, symposia, and workshops are organized in which senior OBS experts from various parts of the globe serve as faculty.

In a similar fashion, it would be pertinent if our colleagues from other oncology and para-clinical disciplines also start integrating OBS principles relevant to their domain in their professional repertoire. We strongly urge oncology-aligned professional societies to incorporate dedicated OBS-themed training sessions in their training workshops, conferences, and symposia.

\section{Conclusions}

OBS in India is currently nascent but expected to attain mainstream status in near future. Taken together, the aforementioned efforts and innovative OBS procedures have revealed the economic feasibility and cost-effectiveness of this novel surgery option in low-resource settings such as India. In general, we have observed that the medical costs to the patients after OBS do not escalate since (a) the same surgeon performs the two necessary roles, (b) operative time is unchanged, (c) second-stage surgery is not needed, (d) surgical accessories (ADMs) are not required, (e) no delay in start of adjuvant treatment, and (f) no specialized RT protocols are required. Indeed, our experiences from the past 5 years from a dedicated breast oncoplastic unit reveal that the OBS hospitalization costs have been reduced by $50 \%$ thereby, leading to high $(80 \%)$ rates of OBS acceptance.

The future of OBS in LMICs such as India is promising but also challenging. As global trends indicate, mainstreaming of OBS in routine breast cancer management in LMICs is inevitable. Young and educated Indian women and their families are increasingly becoming aware of these global developments in OBS. Given the esthetic importance of breasts, issues of self-esteem, body image, and sexual and psychological well-being have started becoming more important in this demography of breast cancer patients. In this regard, the impact of mind-body harmony on cancer treatment outcomes and disease prognosis cannot be over-emphasized. Therefore, it is conceivable that breast surgeons with OBS skills will be preferred by well-informed patients from tier I-III cities and semi-urban localities.

\section{Recommendations}

These stated current and anticipated future circumstances in India provide opportune avenues for all stakeholders in the realm of breast cancer management to innovate and improvise in the larger interest of the patient. This herculean task will require contributions from everyone including the patient (i.e., acceptance of advised OBS options), breast surgeon (i.e., constantly updating oncoplastic surgical skills and knowledge), medical fraternity (i.e., effective integration of domain expertise with OBS principles), medical eco-system (i.e., providing optimal paramedical and infrastructure support), scientific community (i.e., prioritizing innovations that serve OBS needs), insurance sector (i.e., creating OBS coverage policies), government (regulating patient-centric policies and guidelines), patient advocacy groups (i.e., championing patient rights for OBS), and society at large (i.e., sensitizing community about OBS).

We urge our colleagues from various walks of life to dedicate their efforts towards increasing OBS awareness and patient advocacy in there spheres of influence. This could be achieved via various OBS-themed community awareness and outreach campaigns, pink-ribbon support groups, annual marathons, Q\&A sessions with experts, symposia, and joint events with local audio-visual and print media houses.

In summation, we should strive to make it our professional and personal goal to provide affordable excellence in OBS to every breast cancer patient in India regardless of their socioeconomic profile.

Abbreviations $A C M G$, American College of Medical Genetics; $A D M s$, acellular dermal matrix; $A L D S$, autologous lower dermal sling; $B C T$, breast conservation therapy; $C G H S$, Central Government Health Scheme; $E O$, extreme oncoplasty; $H B O C$, hereditary breast and ovarian cancers; $I B R$, immediate breast reconstruction; $L M I C$, low-to-middle income countries; $N A C T$, neo-adjuvant chemotherapy; $N C C N$, National Comprehensive Cancer Network; $O B S$, oncoplastic breast surgery; $P R O M s$, patient-reported outcome measures; $Q o L$, quality of life; $R T$, radiation therapy; $T M$, total mastectomy

\section{References}

1. Strach MC, Prasanna T, Kirova YM, Alran S, O’Toole S, Beith JM, Poortmans P, McNeil CM, Carroll S (2018) Optimise not compromise: the importance of a multidisciplinary breast cancer patient pathway in the era of oncoplastic and reconstructive surgery. Crit Rev Oncol Hematol 134:10-21. https://doi.org/10.1016/j. critrevonc.2018.11.007 
2. Veronesi U, Cascinelli N, Mariani L, Greco M, Saccozzi R, Luini A, Aguilar M, Marubini E (2002) Twenty-year follow-up of a randomized study comparing breastconserving surgery with radical mastectomy for early breast cancer. N Engl J Med 347(16):1227-1232

3. Early Breast Cancer Trialists' Collaborative Group (EBCTCG), McGale P, Taylor C, Correa C, Cutter D, Duane F et al (2014) Effect of radiotherapy after mastectomy and axillary surgery on 10-year recurrence and 20-year breast cancer mortality: metaanalysis of individual patient data for 8135 women in 22 randomised trials. Lancet. 383(9935):2127-2135

4. Gu J, Groot G, Boden C, Busch A, Holtslander L, Lim H (2018) Review of factors influencing women's choice of mastectomy versus breast conserving therapy in early stage breast cancer: a systematic review. Clin Breast Cancer 18(4):e539-e554

5. Waljee JF, Hu ES, Ubel PA, Smith DM, Newman LA, Alderman AK (2008) Effect of esthetic outcome after breast-conserving surgery on psychosocial functioning and quality of life. J Clin Oncol 26(20):3331-3337

6. Macmillan RD, McCulley SJ (2016) Oncoplastic breast surgery: what, when and for whom? Curr Breast Cancer Rep 8(2):112-117

7. Lebovic GS (2010) Oncoplastic surgery: a creative approach to breast cancer management. Surg Oncol Clin N Am 19(3):567-580

8. Benson JR, Down SK (2017) Coming of age of oncoplastic breast surgery. Br J Surg 104(10):1269-1271

9. Audretsch W, Rezai M, Kolotas C (1998) Tumour-specific immediate reconstruction in breast cancer patients. Semin Plast Surg 11:71-99

10. Weber WP, Soysal SD, Fulco I, Barandun M, Babst D, Kalbermatten D, Schaefer DJ, Oertli D, Kappos EA, Haug M (2017) Standardization of oncoplastic breast conserving surgery. Eur J Surg Oncol 43(7):1236-1243

11. van Paridon MW, Kamali P, Paul MA, Wu W, Ibrahim AMS, Kansal KJ, Houlihan MJ, Morris DJ, Lee BT, Lin SJ, Sharma R (2017) Oncoplastic breast surgery: achieving oncological and aesthetic outcomes. J Surg Oncol 116(2):195-202

12. Chatterjee A, Gass J, Burke MB, Kopkash K, El-Tamer MB, Holmes DR et al (2018) Results from the American Society of Breast Surgeons Oncoplastic Surgery Committee 2017 Survey: current practice and future directions. Ann Surg Oncol 24(4):1-5

13. Challoner T, Skillman J, Wallis K, Vourvachis M, Whisker L, Hardwicke J (2017) Oncoplastic techniques: attitudes and changing practice amongst breast and plastic surgeons in Great Britain. Breast 34:58-64

14. Association of Breast Surgery at BASO, Association of Breast Surgery at BAPRAS, Training Interface Group in Breast Surgery, Baildam A, Bishop H, Boland G et al (2007) Oncoplastic breast surgery-a guide to good practice. Eur J Surg Oncol 33 Suppl 1:S1-S23

15. Clough KB, Ihrai T, Oden S, Kaufman G, Massey E, Nos C (2012) Oncoplastic surgery for breast cancer based on tumour location and a quadrant-perquadrant atlas. Br J Surg 99(10):1389-1395

16. Nahabedian MY, Momen B, Galdino G, Manson PN (2002) Breast reconstruction with the free TRAM or DIEP flap: patient selection, choice of flap, and outcome. Plast Reconstr Surg 110(2):466-475

17. Urban C, Anselmi KF, Kuroda F, Schwartz J-C et al (2014) Oncoplasty as the standard of care in breast cancer surgery. Eur Oncol Haematol 10(01):43-45

18. The European Society of Surgical Oncology (2016) ESSO advanced course on oncoplastic breast surgery, Budapest, $p$ 1-9

19. Bray F, Ferlay J, Soerjomataram I, Siegel R, Jemal A (2018) GLOBOCAN estimates of incidence and mortality worldwide for 36 cancers in 185 countries. CA Cancer J Clin 68(6):394-424

20. Freitas-Junior R, Faria SS, Paulinelli RR, Martins E (2017) Trends in oncoplastic breast surgery and breast reconstruction over the past 35 years. Breast J 13(6): 145

21. Silverstein MJ, Savalia N, Khan S, Ryan J (2015) Extreme oncoplasty: breast conservation for patients who need mastectomy. Breast J 21(1):52-59
22. Crown A, Laskin R, Rocha FG, Grumley J (2019) Extreme oncoplasty: expanding indications for breast conservation. Am J Surg. https://doi.org/10.1016/j.amjsurg.2019.01.004

23. Koppiker CB, Noor AU, Dixit S, Busheri L, Sharan G, Dhar U, Allampati HK, Nare S (2019) Extreme Oncoplastic surgery for multifocal/multicentric and locally advanced breast cancer. Int $\mathrm{J}$ Breast Cancer 2019:4262589. https://doi.org/10.1155/2019/ 4262589

24. Piper ML, Esserman LJ, Sbitany H, Peled AW (2016) Outcomes following oncoplastic reduction mammoplasty: a systematic review. Ann Plast Surg 76(Suppl 3):S222-S226

25. Crown A, Handy N, Rocha FG, Grumley JW (2018 May) Oncoplastic reduction mammaplasty, an effective and safe method of breast conservation. Am J Surg 215(5):910-915

26. D'souza N, Darmanin G, Fedorowicz Z (2011) Immediate versus delayed reconstruction following surgery for breast cancer. Cochrane Database Syst Rev 1(7):CD008674

27. O'Halloran N, Potter S, Kerin M, Lowery A (2018 Aug) Recent advances and future directions in postmastectomy breast reconstruction. Clin Breast Cancer 18(4):e571-e585

28. Becker H, Lind JG (2013) The use of synthetic mesh in reconstructive, revision, and cosmetic breast surgery. Aesthet Plast Surg 37(5): 914-921

29. Crisera CA, Chang EI, Da Lio AL, Festekjian JH, Mehrara BJ (2011) Immediate free flap reconstruction for advanced-stage breast cancer: is it safe? Plast Reconstr Surg 128(1):32-41

30. Koppiker CB, Noor AU, Dixit S, Mahajan R, Sharan G, Dhar U et al (2019) Implant-based breast reconstruction with autologous lower dermal sling and radiation therapy outcomes. Indian J Surg. https://doi.org/10.1007/s12262-018-1856-7

31. Cordeiro PG, Albornoz CR, McCormick B, Hu Q, Van Zee K (2014) The impact of postmastectomy radiotherapy on two-stage implant breast reconstruction: an analysis of long-term surgical outcomes, aesthetic results, and satisfaction over 13 years. Plast Reconstr Surg 134(4):588-595

32. Chun YS, Verma K, Rosen H, Lipsitz S, Morris D, Kenney P, Eriksson E (2010) Implant-based breast reconstruction using acellular dermal matrix and the risk of postoperative complications. Plast Reconstr Surg 125(2):429-436

33. Ward RC, Wiggins DL, Donegan L, Collins S, Lourenco AP, Mainiero MB (2018) BioZorb ${ }^{\circledR}$ tissue marker as seen on multiple imaging modalities. Breast J 24(2):207-209

34. National Comprehensive Cancer Network (2018) Breast cancer, $1 \mathrm{st}$ edn, pp 1-209

35. Ludwig KK, Neuner J, Butler A, Geurts JL, Kong AL (2016) Risk reduction and survival benefit of prophylactic surgery in BRCA mutation carriers, a systematic review. Am J Surg 212(4):660-669

36. Saini KS, Taylor C, Ramirez A-J, Palmieri C, Gunnarsson U, Schmoll HJ, Dolci SM, Ghenne C, Metzger-Filho O, Skrzypski M, Paesmans M, Ameye L, Piccart-Gebhart MJ, de Azambuja E (2012) Role of the multidisciplinary team in breast cancer management: results from a large international survey involving 39 countries. Ann Oncol 23(4):853-859

37. Volders JH, Haloua MH, Krekel NMA, Negenborn VL, Barbé E, Sietses C, Jóźwiak K, Meijer S, van den Tol M, "the nationwide network and registry of histo- and cytopathology in the Netherlands (PALGA)" Neoadjuvant chemotherapy in breast-conserving surgery - consequences on margin status and excision volumes: a nationwide pathology study. Eur J Surg Oncol 2016;42(7):986-993

38. Nelson JA, Disa JJ (2017 Nov) Breast reconstruction and radiation therapy: an update. Plast Reconstr Surg 140(5S Advances in Breast Reconstruction):60S-68S

39. La Cruz De L, Blankenship SA, Chatterjee A, Geha R, Nocera N, Czerniecki BJ et al (2016) Outcomes after oncoplastic breastconserving surgery in breast cancer patients: a systematic literature review. Ann Surg Oncol 23(10):3247-3258 
40. Pusic AL, Klassen AF, Scott AM, Klok JA, Cordeiro PG, Cano SJ (2009) Development of a new patient-reported outcome measure for breast surgery: the BREAST-Q. Plast Reconstr Surg 124(2): 345-353

41. Shekhawat L, Dixit S, Busheri L, Patel C, Dhar U, Koppiker CB (2015) Patient-reported outcomes following breast reconstruction surgery and therapeutic mammoplasty: prospective evaluation 1 year post-surgery with BREAST-Q questionnaire. Indian J Surg Oncol 6:356-362. https://doi.org/10. 1007/s13193-015-0432-x,2015
42. Zucca Matthes AG, Viera RADC, Michelli RAD, Ribeiro GHFP, Bailão A, Haikel RL et al (2012) The development of an oncoplastic training center - OTC. Int J Surg 10(5):265-269

43. Maxwell J, Arnaout A, Hanrahan R, Brackstone M (2017) Training oncoplastic breast surgeons: the Canadian fellowship experience. Curr Oncol 24(5):e394-e402

Publisher's Note Springer Nature remains neutral with regard to jurisdictional claims in published maps and institutional affiliations. 